\title{
Drug Reaction with Eosinophilia and Systemic Symptoms (DRESS) with Teicoplanin: A Case Report
}

\author{
Sholeh Ebrahimpour ${ }^{1} \cdot$ Mehdi Mohammadi $^{1} \cdot$ Kheirollah Gholami $^{1,2}$
}

Published online: 20 December 2016

(C) The Author(s) 2016. This article is published with open access at Springerlink.com

\begin{abstract}
Intramuscular teicoplanin (400 mg every $12 \mathrm{~h}$ for three doses, then $400 \mathrm{mg}$ daily, intramuscularly) was prescribed for a 37-year-old woman with presumptive diagnosis of cellulitis. On the 14th day of treatment, she developed generalized maculopapular rash, accompanied by fever, wheezing, shortening of breath, and lymphadenopathy. Lab tests revealed abnormal liver enzymes, leukocytosis, and eosinophilia. The treatment was interrupted with suspicion of drug reaction. Fever subsided after $48 \mathrm{~h}$. Skin eruption and respiratory symptoms began to resolve within 2 weeks. The follow-up lab tests performed 1 month later indicated resolution of liver dysfunction. With respect to delayed onset of symptoms including fever, generalized rash, lymphadenopathy, and organ involvement, drug reaction with eosinophilia and systemic symptoms (DRESS) was highly suspected. The causality was evaluated by conventional scoring systems. The reaction was rated as probable (score $=5$ ) according to RegiSCAR and possible (score $=5$ ) based on Kardaun et al.'s scoring system. However, DRESS was not confirmed by the Japanese group's criteria for diagnosis of DRESS/druginduced hypersensitivity syndrome (DIHS).
\end{abstract}

Kheirollah Gholami

khgholami@tums.ac.ir

1 Clinical Pharmacy Department, Faculty of Pharmacy, Tehran University of Medical Sciences, Tehran, Iran

2 Research Center for Rational Use of Drugs, Tehran University of Medical Sciences, 4th Floor, No. 92, Karimkhan Zand Avenue, Hafte Tir Square, Tehran, Iran

\section{Key Points}

Drug reaction with eosinophilia and systemic symptoms (DRESS) is a life-threatening reaction that necessitates determination and discontinuation of the offending drug.

The aromatic structure of teicoplanin is shared by most other medications involved in DRESS.

The use of additional treatment including intravenous immunoglobulins, corticosteroids and antivirals is generally based on experience rather than proven benefits drawn from well-designed clinical trials.

\section{Introduction}

Drug reaction with eosinophilia and systemic symptoms (DRESS) syndrome is defined as an idiosyncratic, rare, and life-threatening reaction. The clinical features of the syndrome, including fever, rash, facial edema, lymphadenopathy, hematological abnormality, and internal organ involvement, arise 10-30 days following drug exposure. This late onset of symptoms discriminates DRESS from some other drug-induced skin reactions such as erythema morbilliform $[1,2]$. The most common suspected medicines causing DRESS include aromatic anticonvulsants (carbamazepine, phenytoin, phenobarbital, and lamotrigine), allopurinol, and antibiotics (sulfasalazine, vancomycin, and minocycline) [2]. To the best of our knowledge, there are limited reports of teicoplanin-induced DRESS in the literature [2-6]. Here, we report a case of 
DRESS associated with teicoplanin. This report is important to enhance our knowledge on severe side effects of teicoplanin.

\section{Case Report}

A 37-year-old woman was admitted to hospital with redness and edema of inguinal area. The involved area was tender and warm on examination. With a presumptive diagnosis of cellulitis, vancomycin $1 \mathrm{~g}$ twice daily was prescribed. After $24 \mathrm{~h}$, due to the acceptable clinical state of the patient, treatment was planned to be completed in the ambulatory setting. Vancomycin was replaced with teicoplanin, considering its ease of administration as an intramuscular injection (400 mg every $12 \mathrm{~h}$ for three doses, then $400 \mathrm{mg}$ daily).

On the 14th day of treatment, the patient developed generalized maculopapular rash (Fig. 1), accompanied by fever $\left(39^{\circ} \mathrm{C}\right)$, wheezing, shortening of breath, and cervical and axillary lymphadenopathy. Lab tests revealed abnormal liver enzymes [alanine aminotransferase (ALT) 134 IU/L, aspartate transaminase (AST) 141 IU/L, alkaline phosphatase (ALP) $345 \mathrm{IU} / \mathrm{L}$, leukocytosis (white blood cell count $17,000 / \mu \mathrm{L}$ ) with eosinophilia to more than $8 \%(1360 / \mu \mathrm{L})$, a blood urea nitrogen $(\mathrm{BUN})$ value of $24 \mathrm{mg} / \mathrm{dL}$, and a serum creatinine (SCr) value of $0.8 \mathrm{mg} /$ $\mathrm{dL}$. The treatment was interrupted with suspicion of drug reaction. After $48 \mathrm{~h}$, the patient defervesced. Skin eruption and respiratory symptoms began to resolve within 2 weeks. The follow-up lab test performed 1 month later indicated resolution of liver dysfunction (ALT $22 \mathrm{U} / \mathrm{L}$, AST $18 \mathrm{U} / \mathrm{L})$.

\section{Discussion}

With respect to diversity in scoring systems and differential diagnoses, the exact incidence of DRESS, as a life-threatening skin reaction, remains unknown. This could be partially because there is no gold-standard test for diagnosis of DRESS, and as a result, the diagnosis remains a challenge and is mainly based on conventional proposed scoring systems. The most common scoring systems to stratify DRESS are RegiSCAR [7], the Japanese group's criteria for diagnosis of DRESS/drug-induced hypersensitivity syndrome (DIHS) [8], and a system proposed by Kardaun et al. [9] (Table 1).

DRESS is classified as a type IV drug-induced hypersensitivity reaction that is characterized by delayed onset of symptoms. The rising of eosinophil count and non-necrotizing lesions differentiate DRESS from other type IV drug-induced hypersensitivity reactions such as StevensJohnson syndrome/toxic epidermal necrolysis (SJS/TEN). In regard to delayed onset of signs and symptoms including skin rash (more than $50 \%$ of body surface area), fever (more than $38.5^{\circ} \mathrm{C}$ ), and enlarged lymph node (more than $1 \mathrm{~cm}$ in two sites), DRESS was highly suspected. These findings are in concordance with previous reports of teicoplanin-induced DRESS [3, 4, 6]. Additional work-up was performed to evaluate hematological abnormalities and organ involvement, which revealed leukocytosis with eosinophilia and liver involvement.

It is noticeable that the patient work-up remained incomplete. Chest x-ray or computerized tomography (CT) scan and skin biopsy were not performed due to patient non-compliance. Therefore, pulmonary involvement was judged only on the basis of clinical symptoms. The
Fig. 1 Generalized maculopapular rash on the neck and trunk

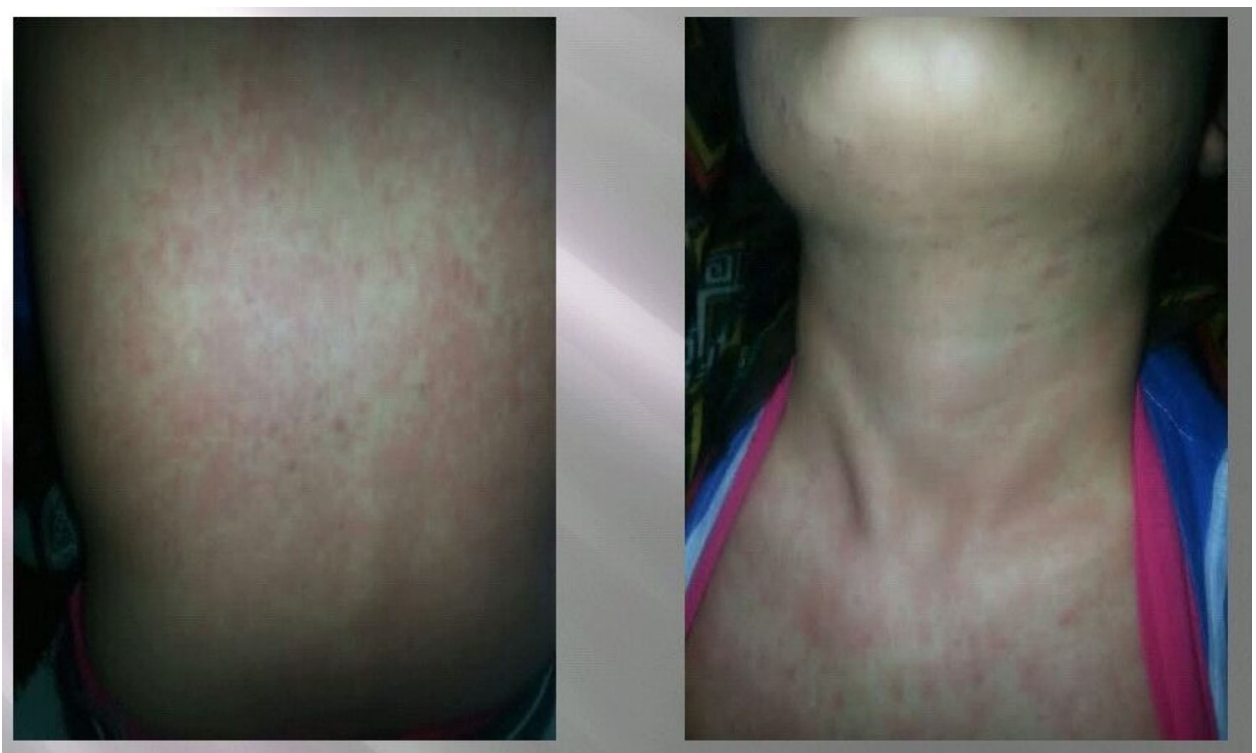


Table 1 Kardaun et al.'s scoring system [9] Reproduced from Kardaun et al. [9], with permission

\begin{tabular}{llrl}
\hline Item & Present & $\begin{array}{c}\text { Absent } \\
\text { Patient's } \\
\text { score }\end{array}$ \\
\hline Fever $\geq 38.5^{\circ} \mathrm{C}\left(101.3^{\circ} \mathrm{F}\right)$ & 0 & -1 & 0 \\
Enlarged lymph nodes $(>1 \mathrm{~cm}$ size, at least two sites) & 1 & 0 & 1 \\
Eosinophilia: $\geq 700$ or $\geq 10 \%$ (leucopenia); $\geq 1500$ or $\geq 20 \%$ (leucopenia) & $1 ; 2$ & 0 & 1 \\
Atypical lymphocytes & 1 & 0 & 0 \\
Rash $\geq 50 \%$ of body surface area & 1 & 0 & 1 \\
Rash suggestive $(\geq 2$ of facial edema, purpura, infiltration, desquamation) & 1 & 0 & 0 \\
Skin biopsy suggesting alternative diagnosis & -1 & 0 & 0 \\
Organ involvement: $1 ; \geq 2$ & $1 ; 2$ & 0 & 2 \\
Disease duration $>15$ days & 0 & -2 & 0 \\
Investigation for alternative cause (blood cultures, ANA*, serology for & 1 & 0 & 0 \\
hepatitis viruses, mycoplasma, Chlamydia), $\geq 3$ done and negative & & & \\
Total score & & & 5 \\
\hline
\end{tabular}

Total score $<2$ : excluded; $2-3$ : possible; $4-5$ : probable; $\geq 6$ : definite

*ANA antinuclear antibody association of viral reactivation with DRESS has been reported in previous publications [10]. Testing for human herpesvirus-6, human herpesvirus-7, and Epstein-Barr virus antibodies was not requested because of limited resources. In general, our presumptive diagnosis was mainly based on clinical signs and symptoms and accessible lab tests.

On the basis of the scoring systems mentioned above, the reaction was rated as probable ( RegiSCAR and possible (score $=5$ ) according to Kardaun et al.'s scoring system. Since presence of atypical lymphocytes and reactivation of human herpesvirus were not investigated, DRESS was not confirmed by the Japanese group's criteria for diagnosis of DRESS/DIHS.

Regardless of the aforementioned limitations, the clinical picture was in favor of DRESS.

Anticonvulsants with aromatic structure are the most common agents associated with DRESS. The aromatic structure of vancomycin and teicoplanin may explain the occurrence of DRESS with these agents [2].

In this case, teicoplanin was used instead of vancomycin according to the Summary of Product Characteristics (available at http://www.sanofi.com.au). Given the similar structure of vancomycin and teicoplanin, cross-reactivity is anticipated. Therefore, vancomycin may have prompted the reaction with teicoplanin. Resolution of symptoms after discontinuation of teicoplanin highlights it as the causative agent.

Withdrawal of the offending medication and supportive care are the mainstay of management. The implementation of additional treatment including intravenous immunoglobulins, corticosteroids and antivirals is generally based on experience rather than proven benefits drawn from well-designed clinical trials [11-13]. Administration of corticosteroids in severe pulmonary involvement seems to be reasonable according to results from some studies $[13,14]$. However, we did not implement these interventions, considering the lack of proven benefit and the patient's overall state of health. Furthermore, without performing imaging tests, pulmonary involvement could not be confirmed.

Acknowledgements We would like to thank Dr. Hamid-reza Taghva Masoumi for his expert advice and encouragement through this work.

\section{Compliance with Ethical standards}

Written informed consent was obtained from the patient for publication of this case report and any accompanying images. A copy of the written consent may be requested for review from the corresponding author.

Conflicts of interest Sholeh Ebrahimpour, Mehdi Mohammadi, and Kheirollah Gholami declare that they have no conflicts of interest relating to the content of this article.

Author contributions SE performed patient follow-up and prepared the initial manuscript. $\mathrm{KH}$ and $\mathrm{MM}$ contributed to interpretation of data and manuscript revision.

Open Access This article is distributed under the terms of the Creative Commons Attribution-NonCommercial 4.0 International License (http://creativecommons.org/licenses/by-nc/4.0/), which permits any noncommercial use, distribution, and reproduction in any medium, provided you give appropriate credit to the original author(s) and the source, provide a link to the Creative Commons license, and indicate if changes were made.

\section{References}

1. Choudhary S, McLeod M, Torchia D, Romanelli P. Drug reaction with eosinophilia and systemic symptoms (DRESS) syndrome. J Clin Aesthet Dermatol. 2013;6(6):31-7. 
2. Renda F, Landoni G, Bertini Malgarini R, Assisi A, Azzolini ML, Mucchetti M, et al. Drug reaction with eosinophilia and systemic symptoms (DRESS): a national analysis of data from 10-year post-marketing surveillance. Drug Saf. 2015;38(12):1211-8.

3. Tamagawa-Mineoka R, Katoh N, Nara T, Nishimura Y, Yamamoto S, Kishimoto S. DRESS syndrome caused by teicoplanin and vancomycin, associated with reactivation of human herpesvirus-6. Int J Dermatol. 2007;46(6):654-5.

4. Loussaief C, Kooli I, Ben Fradj N, Ben Brahim H, Aouam A, Toumi A, et al. Note technique DRESS syndrome induit par la teicoplanine. Revue Francaise d'Allergologie. 2015;55(4):308-11.

5. Perrett CM, McBride SR. Teicoplanin induced drug hypersensitivity syndrome. BMJ. 2004;328(7451):1292.

6. Hsiao SH, Chen HH, Chou CH, Lin WL, Liu Yeh PY, Wu TJ. Teicoplanin-induced hypersensitivity syndrome with a preceding vancomycin-induced neutropenia: a case report and literature review. J Clin Pharm Ther. 2010;35(6):729-32.

7. Roujeau J-C, Allanore L, Liss Y, Mockenhaupt M. Severe cutaneous adverse reactions to drugs (SCAR): definitions, diagnostic criteria, genetic predisposition. Dermatol Sinica. 2009;27:203-9.

8. Shiohara T, Inaoka M, Kano Y. Drug-induced hypersensitivity syndrome (DIHS): a reaction induced by a complex interplay among herpesviruses and antiviral and antidrug immune responses. Allergol Int Off J Jpn Soc Allergol. 2006;55(1):1-8.
9. Kardaun SH, Sidoroff A, Valeyrie-Allanore L, Halevy S, Davidovici BB, Mockenhaupt $\mathrm{M}$, et al. Variability in the clinical pattern of cutaneous side-effects of drugs with systemic symptoms: does a DRESS syndrome really exist? Br J Dermatol. 2007;156(3):609-11.

10. Tohyama M, Hashimoto K, Yasukawa M, Kimura H, Horikawa T, Nakajima K, et al. Association of human herpesvirus 6 reactivation with the flaring and severity of drug-induced hypersensitivity syndrome. Br J Dermatol. 2007;157(5):934-40.

11. Joly P, Janela B, Tetart F, Rogez S, Picard D, D'Incan M, et al. Poor benefit/risk balance of intravenous immunoglobulins in DRESS. Arch Dermatol. 2012;148(4):543-4.

12. Chen YC, Chiu HC, Chu CY. Drug reaction with eosinophilia and systemic symptoms: a retrospective study of 60 cases. Arch Dermatol. 2010;146(12):1373-9.

13. Descamps V, Ben Said B, Sassolas B, Truchetet F, Avenel-Audran M, Girardin P, et al. Management of drug reaction with eosinophilia and systemic symptoms (DRESS). Ann Dermatol Venereol. 2010;137(11):703-8.

14. Sultan SJ, Sameem F, Ashraf M. Drug reaction with eosinophilia and systemic symptoms: manifestations, treatment, and outcome in 17 patients. Int J Dermatol. 2015;54(5):537-42. 